\title{
Experimental study of equivalence ratio and fuel flow rate effects on nonlinear thermoacoustic instability in a swirl combustor
}

\author{
He Zhao ${ }^{\mathrm{a}}$, Guoneng $\mathrm{Li}^{\mathrm{b}}$, Dan Zhao ${ }^{\mathrm{c}}$, , Zhiguo Zhang ${ }^{\mathrm{b}}$, Dakun Sun ${ }^{\mathrm{d}}$, Wenming Yang ${ }^{\mathrm{e}}$, Shen $\mathrm{Li}^{\mathrm{a}}$, Zhengli Lu ${ }^{\mathrm{a}}$, \\ Youqu Zheng ${ }^{\mathrm{b}}$ \\ a School of Energy and Power Engineering, Jiangsu Univeristy of Science and Technology, Zhejiang City, Mengxi Road 2, Jiangsu Province 212003, China \\ b School of Mechanical and Automotive Engineering, Zhejiang University of Science and Technology, Hangzhou City, Liuhe Road, Zhejiang 310023, China \\ ${ }^{c}$ Department of Mechanical Engineering, College of Engineering, University of Canterbury, Private Bag 4800, Christchurch 8140, New Zealand \\ d Co-Innovation Center for Advanced Aero-engine, Beijing University of Aeronautics and Astronautics, Beijing 100191, China \\ ${ }^{\mathrm{e}}$ Department of Mechanical Engineering, Faculty of Engineering, National University of Singapore, 9 Engineering Drive, Singapore 117576, Singapore
}

\section{ART ICLE INFO}

\section{Keywords:}

Thermoacoustics

Mode switching

Swirl combustor

Combustion instability

Recurrence analysis

Energy conversion

\begin{abstract}
A B S T R A C T
Industrial combustion systems such as power generation gas turbines, rocket motors, furnaces and boilers often face the problem of large-amplitude self-excited pressure oscillations that occur due to the onset of thermoacoustic instability. To prevent the onset of such instability, understanding the effects of fuel-air equivalence ratio $\phi$, fuel flow rate $\mathrm{V}_{\mathrm{f}}$ on self-excited nonlinear thermoacoustic oscillations is of fundamental and practical importance. Experimental investigation of the roles of these parameters on triggering thermoacoustic instability in a swirl combustor has received very little attention. In this work, we design a swirling thermocoustic combustor and conduct a series of experimental tests. Autocorrelation and recurrence analysis of phase space trajectories reconstructed from the acoustic pressure time trace are performed. These experimental tests allow us to study the effect of fuel-air equivalence ratio $\phi$ on the onset of thermoacoustic instability by varying the fuel volume flow rate $V_{f}$. We demonstrate that the fuel volume flow rate and the equivalence ratio play different but critical roles on generating thermoacoustic instability at different frequencies and amplitudes. Maximum sound pressure level can be as high as $135 \mathrm{~dB}$. In addition, mode switching, (i.e. frequency swap) is found to occur between approximately $\omega_{3} \approx 510 \mathrm{~Hz}$ and $\omega_{1} \approx 170 \mathrm{~Hz}$, depending on the equivalence ratio $\phi$. Furthermore, the dominant frequency corresponding to the maximum amplitude is shown to be shifted by approximately $20 \%$, as the fuel flow rate $V_{f}$ is increased and the combustion condition is changed from lean to rich. These findings are quite useful for designing a feedback control strategy to stabilize an unstable combustor. The present work opens up an applicable means to design a stable swirling combustor.
\end{abstract}

\section{Introduction}

Self-excited thermoacoustic oscillations occur in many systems such as thermoacoustic standing- or travelling-wave heat engines [1-3], industrial furnaces, gas turbines and thermoacoustic refrigerators [4-7]. The phenomenon results from a feedback loop between unsteady heat release process $[8,9]$ and acoustic perturbations [10]. The heat release response to acoustic disturbances is generally nonlinear [11]. As a critical operating parameter such as the fuel flow rate is varied, the onset of self-sustained thermoacoustic oscillations occurs via a supercritical (soft) or subcritical (hard) Hopf bifurcation [9,12,13]. In the case of a subcritical Hopf bifurcation, a bi-stable region exists for a range of the parameter values. This region is associated with nonlinear phenomena such as triggering and hysteresis [12,13].
The occurrence of detrimental thermoacoustic instability in lean, premixed combustors hinders the development of modern gas turbine engines [2] for aerospace and power generation industries. These instabilities are characterized by large-amplitude, periodic oscillations of the combustor's acoustic fields [3]. These oscillations are undesirable, since they significantly reduce the lifetime and regions of operability of the engine combustor. To mitigate these instabilities [6], a number of experimental and numerical studies on the generation mechanisms and approaches for its control have been conducted during the past few decades. Several of these investigations have confirmed that these instabilities are most likely produced by a mechanism involving the interactions between heat release perturbations, acoustic pressure disturbances, and reactive mixture composition fluctuations [12].

Previous studies revealed that an understanding of the mechanism that is responsible for generating thermoacoustic instability does not necessarily

\footnotetext{
* Corresponding author.

Email address: dan.zhao@canterbury.ac.nz (D. Zhao)
} 
vide any insightful information on the process that determines the nonlinear oscillations of the combustion system. Such an understanding of the transient and steady state nonlinear oscillating characteristics of an unstable combustor is needed so that the amplitude of the oscillations is predictable or feedback control approaches [3] are developed and optimized to stabilize unstable combustion systems [6]. The role of the nonlinear processes occurred in the combustors on initiating and sustaining thermoacoustic instabilities has been and continues to be a subject of intensive and extensive research. A great deal of physical insights into the complex nonlinear behaviors that is often observed in unstable premixed $[5,9,10]$ or swirl combustors [4] are obtained. For example, it was demonstrated the existence of stable limit cycles in unstable combustors or the possibility of 'triggering' of such instability in a linearly stable system $[12,13]$.

Combustion systems involve complex coupling between hydrodynamics, acoustics and combustion process $[5,10]$. In the past few decades, statistical analyses of the time series measurements and frequency-domain analysis of combustion systems are extensively performed [5]. Recently, there are strong interests in applying recurrence-related methods [14-17] on analyzing unstable combustion dynamics. Recurrence analysis has great potential to be applied to different nonlinear combustion systems to characterize and identify the dynamical similarities between these systems. More importantly the recurrence analysis [14] and related methods $[15,16]$ can provide more extract insightful information from experimental data, which may be neglected due to the observation of very irregular or noisy measurements [18]. A better understanding of combustion dynamics and an improved ability to prevent or mitigate thermoacoustic instability will safely expand the operating envelope of combustors towards a regime that is associated with increased combustion efficiency and decreased chemical emission. This will also help to achieve the objectives of reducing NOx and controlling noise emissions [18-20].

Previous nonlinear time series measurements on combustion dynamics $[21,22]$ have provided several important results in the past. Fractal analysis of time series experimental measurements of combustion noise has been explored by Strahle et al. [21-23]. They found that such nonlinear analysis can provide more meaningful and insightful information from the very noisy measurements. Furthermore, fractal characterization of time series experimental data yielded quantitative measures. These measures could be used to characterize the dynamics behaviors of the combustion system and could thus be used for modelling, analysis and validation. The scientific study on turbulent combustion noise followed the previous researches on turbulent flows and flames [24,25]. Nonlinear means based on the phase space characterization $[26,27]$ are increasingly being combined in investigations on laminar [28] and turbulent combustion [29-34], internal combustion engines [35] as well as related fields [36,37]. These techniques allow studies through an alternate perspective: the phase space. For this, they have been quite successful in gaining more additional insights into the system's dynamics from the time series experimental measurements [38].

In this work, we experimentally study and analyze pressure data measured in an unstable swirl combustor in an effort to gain insights on the nonlinear dynamics characteristics of the thermoacoustic system. In Section 2, the experimental setup, data acquisition and measurement system are described. To monitor the flow fluctuations and temperature, a dynamic pressure sensor and an infrared thermal camera are applied. The measured pressure signal is processed by applying autocorrelation, as described in Section 3. Furthermore, recurrence analysis of the phase space trajectories reconstructed from the acoustic pressure time trace are conducted. In Section 4, two critical parameters of thermoacoustic systems with methane gas burned are studied. One is the fuel volume flow rate and the other is the fuel-air equivalence ratio $\phi$. The effect of the fuel volume flow rate on generating thermoacoustic instability in the swirling thermoacoustic system is studied first by setting it to 4 different values. For each given fuel volume flow rate, the fuel-air equivalence ratio $\Phi$ is changed from 0.8 to 1.2 with an increment step of 0.1 . The key findings are summarized in Section 5 .

\section{Description of experiments}

Experimental tests on a laboratory-scale swirl combustor with a dynamic pressure sensor implemented are performed. The experimental setup is shown in Fig. 1. A premixed methane-fueled flame is enclosed in the bottom half of the cylindrical tube. The burner is anchored on a workbench. The fuel volume flow rate $\mathrm{V}_{\mathrm{f}}$ is variable. A central pilot jet is added. Methane $\mathrm{CH}_{4}$ and air are used as the fuel and oxidizer. The mass flow rates are controlled precisely by two ALICAT and one D07 mass flow controllers (Cs). The accuracy of the MFC is less than $\pm 1.5 \%$. At the inlet, there is a swirler to create swirling flow. The degree of swirl is typically characterized by a non-dimensional swirl number $\mathrm{S}_{\mathrm{w}}$. It is mathematically defined as $S_{w}=\frac{\int_{0}^{R} u_{z} u_{\theta} r^{2} d r}{R \int_{0}^{R} u_{z}^{2} r d r}$. The swirl number denotes the axial flux of swirl momentum with respect to the product of the axial flux of axial momentum and the burner nozzle equivalent radius [39]. $\mathrm{S}_{\mathrm{w}}$ is approximated by its geometric dimensions as $S_{w} \approx \frac{2}{3} \tan \alpha \approx 0.72$ and $\alpha=47$ degrees in this work.

The swirling thermoacoustic combustor is made of stainless steel. The outer diameter and length of the combustor is $71 \mathrm{~mm}$ and $810 \mathrm{~mm}$, as shown schematically in Appendix A. The fuel and air supplying system consists of 4 main components: (1) a fuel supplying nozzle, (2) a straight primary air orifice, (3) three tangential swirling secondary flow pipes and (4) a secondary fuel injector. The fuel supplying nozzle is a round tube with an inner diameter of $6 \mathrm{~mm}$ and an outer diameter of $8 \mathrm{~mm}$. The primary air supplying orifice is installed surrounding the fuel supplying orifice with an inner diameter of $12 \mathrm{~mm}$. Swirling air is forced into join the primary air stream through the three tangential air supplying inlets. Each air supplying inlet is with an inner diameter of $4 \mathrm{~mm}$. The tangential air flow pipes are fixed $60 \mathrm{~mm}$ upstream of the bottom end of the combustor. They are inclined 15 degree upward to the horizontal plane. The central secondary fuel supply pipe has an inner diameter of $2 \mathrm{~mm}$ with an outer diameter of $3 \mathrm{~mm}$.

The acoustic pressure is measured by using a CGY dynamic pressure sensor and logged by National Instruments (NI-PXIe1062Q chassis, PXI4498 sound module). The accuracy and dynamic range of the pressure sensor is $\pm 0.5 \%$ F. S. and $20 \mathrm{kHz}$. It is placed at $710 \mathrm{~mm}$ away from the top open end of the tube, with a semi-infinite technique used to obtain thermal insulation without distortion from acoustic reflections. The acoustic pressure perturbations are acquired with a sampling rate of 10,000 samples/second. To protect the pressure sensor, water cooling system is designed and applied. The sensor's sensitivity is $600 \mathrm{~Pa} / \mathrm{V}$. To measure the temperature, an infrared thermal imaging camera (Dali-T8 Model) is applied. Its thermal sensitivity is $\leq 0.05 \mathrm{~K} @ 298 \mathrm{~K}$ and $60 \mathrm{~Hz}$ Fps. The measurement range is $250-2000 \mathrm{~K}$. And the accuracy is $\pm 2 \mathrm{~K}$. For calibration, a K-type thermocouple is also applied.

Equivalence ratio $\Phi$ is a critical parameter indicating combustion conditions such as rich or lean combustion. $\Phi$ denotes the fuel air ratio, which affect the combustion efficiency and thermoacoustic stability $[40,41]$. When stoichiometric combustion (also known as complete combustion) occurs, $\phi=1.0$. Stoichiometric combustion characterizes the condition at which exactly enough air is provided to completely burn all of the fuel to form products (complete combustion). As for the methane is burned and $\phi=1$, the following chemical reaction is assumed to occur:

$\mathrm{CH}_{4}+2\left(\mathrm{O}_{2}+3.76 \mathrm{~N}_{2}\right) \rightarrow \mathrm{CO}_{2}+2 \mathrm{H}_{2} \mathrm{O}+7.52 \mathrm{~N}_{2}$

The composition of air is considered to be $79 \%$ of $\mathrm{N}_{2}$ and $21 \%$ $\mathrm{O}_{2}$ by volume. For each mole of $\mathrm{O}_{2}$ in air, there are $79 / 21(=3.76)$ moles of $\mathrm{N}_{2}$. The ratio 


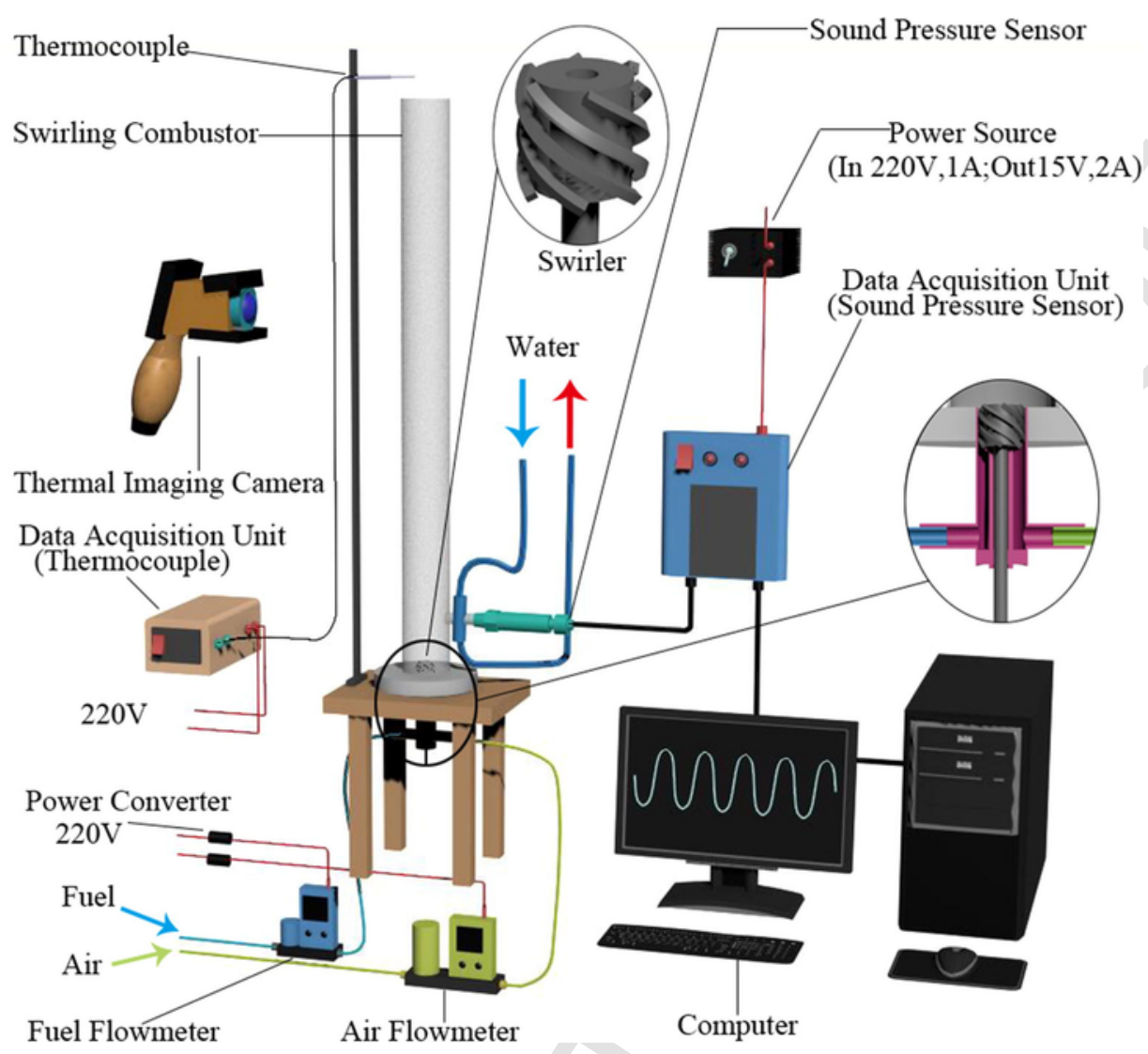

Fig. 1. Schematic of the swirling thermoacoustic combustor.

$\mathrm{V}_{\mathrm{f}} / \mathrm{V}_{\mathrm{a}}$ between the methane and air volume flow rates is then determined as

$\left(\frac{V_{f}}{V_{a}}\right)_{\phi=1}=\frac{1}{2 \times(1+3.76)}=\frac{1}{9.52}=0.105$

In practice, excessive air is typically applied to prevent excessive temperature at the exit of the combustor, and to achieve complete combustion and low combustion emissions. Thus the practical equivalence ratio $\Phi$ is related to the fuel and air volume flow rates as

$\phi=\left(\frac{V_{f}}{V_{a}}\right)_{\text {practice }} /\left(\frac{V_{f}}{V_{a}}\right)_{\phi=1}=\frac{9.52 V_{f}}{V_{a}} \Rightarrow$

It is worth noting that there are NOx and CO produced from practical combustion of methane. However, the amount of these combustion products are negligibly small (in ppm). Thus it is reasonable to consider $\mathrm{CO}_{2}$ and $\mathrm{H}_{2} \mathrm{O}$ as the main products and $\mathrm{N}_{2}$ is assumed to not involve in the combustion.

\section{Analyzing methods}

\subsection{Recurrence plot of the measured pressure signal}

It is known that recurrence of states is an important characteristic in dynamical systems such as gas turbine combustors. Recurrence plot

is

a

graphical tool to visualize the recurrence behaviors [28] in the state space:

$$
\begin{aligned}
\mathbf{p}(i) & =\left(p_{i}, p_{i+\tau}, \cdots p_{i+\tau(M-1)}\right), \quad i \\
& =1,2, \ldots N-\tau(M-1)
\end{aligned}
$$

It is lag-reconstructed from a time series $\boldsymbol{p}$. The embedding dimension $M$ is determined by the false nearest neighbor algorithm [35]. And the time delay parameter $\tau$ is estimated with mutual information method [34]. The un-threshold recurrence (UR) plot represents a symmetric distance matrix between the state vectors, i.e. $U R(i, j)=$ $\|\mathbf{p}(i)-\mathbf{p}(j)\|$. The thresholded recurrence plot is mathematically expressed by a symmetric matrix $R(i, j)$ characterizing the proximity of the state vectors. The RP is defined as

$$
R(i, j)=\Theta(\varepsilon-\|\mathbf{p}(i)-\mathbf{p}(j)\|)
$$

where $\varepsilon$ is a predefined threshold. $\boldsymbol{p}(\mathrm{i})$ and $\boldsymbol{p}(\mathrm{j})$ are vectors in the state space and $\Theta$ is the Heaviside function. Recurrence quantification analysis (RQA) [35] can not only characterize small-structures (e.g., small dots, vertical and diagonal lines) in the recurrence plots but also quantify the system chaos-order or chaos-chaos transitions [34,35]. The recurrence quantifiers include (1) determinism, (2) recurrence rate, entropy, and trapping time. More background on the recurrence quantification analysis of a diesel engine or a ducted combustor with a laminar premixed flame can be found in the previous works $[35,28]$. 


\subsection{Root-mean-square of the pressure measurements}

The root-mean-square $\mathrm{p}_{\mathrm{rms}}$ of a pressure measurement vector, $\boldsymbol{p}$, is

$p_{r m s}=\sqrt{\frac{1}{N} \sum_{n=1}^{N}\left|\mathbf{P}_{n}\right|^{2}}$,

Here, the summation is performed along the pressure measurements. $N$ is the total number of the measured samples.

\subsection{Autocorrelation of the measured pressure signal}

The autocorrelation $\langle\mathrm{p}(\mathrm{t}), \mathrm{p}(\mathrm{t}-\tau)\rangle$ between $p(t)$ and $p(t-\tau)$, and $p(t)$ is a stochastic measurement of the signal from the pressure sensor. The formula for the autocorrelation $\langle\mathrm{p}(\mathrm{t}), \mathrm{p}(\mathrm{t}-\tau)\rangle$ for a lag $k=\tau / \Delta t$ is

$$
\begin{aligned}
\langle p(t), p(t-\tau)\rangle & =\langle p(t), p(t-k \Delta t\rangle \\
& =\frac{\frac{1}{N-1} \sum_{t=1}^{N+k}\left(p_{t}-\bar{p}\right)\left(p_{t-k}-\bar{p}\right)}{c_{0}}
\end{aligned}
$$

where $c_{0}$ is the variance of the time series measurement of the pressure signal $\mathrm{p}(\mathrm{t}) . N$ is the total number of the measured samples.

\section{Results and discussion}

The experimental results are summarized by discussing the thermoacoustic instability occurred in the swirling thermoacoustic system, as the volume flow rate of the methane is set to 4 different values and the equivalence ratio $\phi$ is varied from 0.8 to 1.2 . Fig. 2 shows the measured pressure signals from the swirl combustor, as the methane volume flow rate is set to 4 different values.
It can be seen that for a given $\Phi$, increasing the methane volume flow rate $\mathrm{V}_{\mathrm{f}}$ leads to the occurrence of limit cycle (periodic) oscillations. In addition, the pressure signal is strongly oscillating with time. This is most like due to the presence of the swirling flow. The swirling flow can be visualized from the measured temperature contours by using the infrared thermal image camera as shown in Fig. 3.

It is clear that the non-uniform swirling flow leads to the turbulence-shaped temperature contour. Furthermore, as the fuel-air equivalence ratio $\phi$ is increased from 0.8 to 1.2 , the 'hottest' region and maximum temperature are reduced, due to the increased air flow rate. When combustion instability occurs (see Fig. 2(d)), limit cycle oscillations lead to the combusting flow being 'pulsating'. This is clearly observed in Fig. 3(c) from the wavy edge of the hot combustion flow. In addition, the 'hot' outflow is in the conical shape. And the combusting gas are 'lift-off', after flowing out of the top end of the cylindrical tube. This may be due to the swirling effect.

The corresponding frequency spectrum of the measured pressure signals is shown in Fig. 4. It can be seen from Fig. 4(a) and (b) that when $\phi \leq 0.9$, limit cycle oscillations are not apparent as observed in Fig. 4(c)-(e). Furthermore, there are 2 dominant modes. One is the fundamental mode at $\omega_{1}$. The other is its harmonic peak at $\omega_{3}$. The maximum sound pressure levels at $\omega_{1}$ and $\omega_{3}$ are less than $105 \mathrm{~dB}$, as $\phi \leq 0.9$. And they are comparable. However, as $\phi$ is increased from 1.0 to 1.2, the maximum sound pressure level is increased to $135 \mathrm{~dB}$ and then decreased to $127 \mathrm{~dB}$. In addition, as $1.0 \leq \phi \leq 1.1$ and the methane volume flow rate is increased from $V_{\mathrm{f}}=3.0 \mathrm{~L} / \mathrm{min}$ to $\mathrm{V}_{\mathrm{f}}=5.0 \mathrm{~L} / \mathrm{min}$, the dominant peak corresponding to the maximum sound pressure level is changed from $\omega_{3}$ to $\omega_{1}$. This indicates that the swirl combustor is a nonlinear thermoacoustic system, especially at $1.0 \leq \Phi \leq 1.2$ and $\mathrm{V}_{\mathrm{f}} \geq 3.0 \mathrm{~L} /$ min. Finally, as $\phi$ is fixed and $V_{f}$ is increased, the domain frequency, i.e. $\omega_{1}$ is somehow increased. For example, as $\phi=1.0$, as $V_{\mathrm{f}}$ is increased, $\omega_{1}$ is found to increase by $20 \%$ (see Fig. 4(c)). Same shift trend is found to $\omega_{3}$. This is most likely due to increased total heat release $[42,43]$ and mean temperature.

To gain insights on the intensity of the flow fluctuations in the swirl combustor, the root mean square value of the measured pressure is obtained, as shown in Fig. 5. It can be seen that as $V_{f}=5.0 \mathrm{~L} /$ min as shown in Fig. 5(d), the pressure oscillations become more intensified than the other cases. Further-
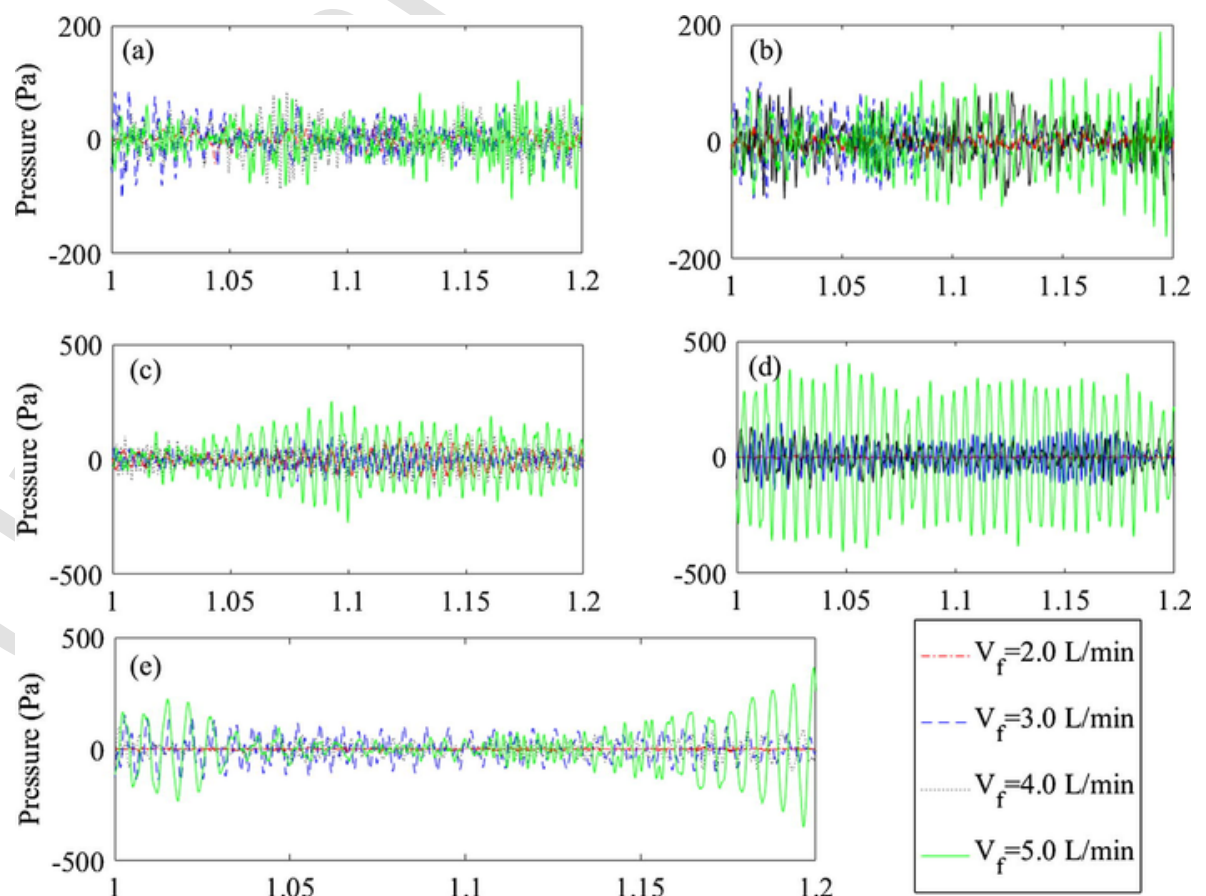

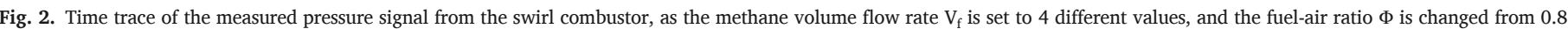
to 1.2 with an increment step of 0.1. (a) $\phi=0.8$, (b) $\phi=0.9$, (c) $\phi=1.0$, (d) $\phi=1.1$ and (e) $\phi=1.2$. 


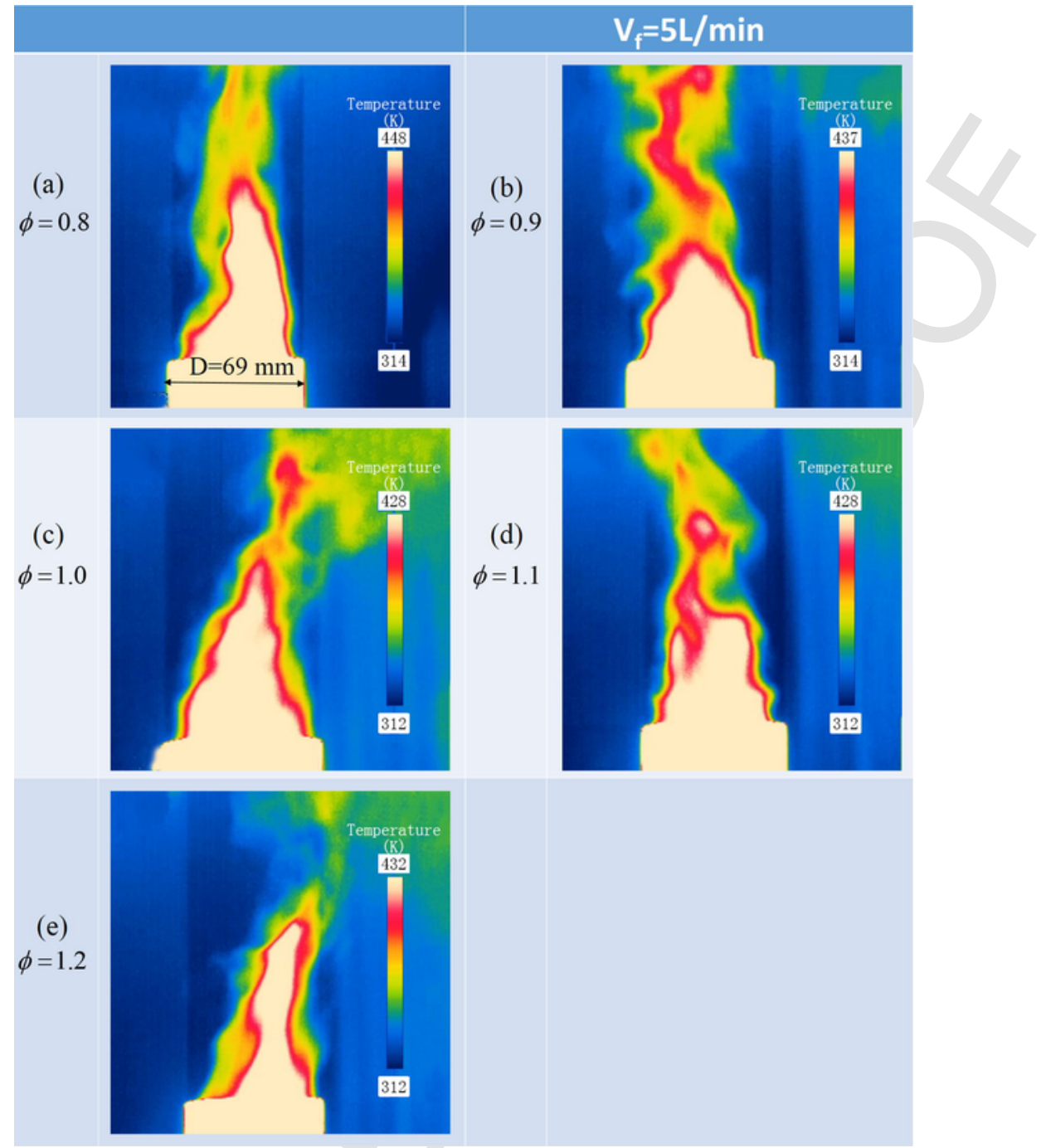

Fig. 3. Measured temperature contours by using the infrared thermal imaging camera, as $\mathrm{V}_{\mathrm{f}}=5.0 \mathrm{~L} / \mathrm{min}$ and $\phi$ is set to 5 different values.

more, as fuel-air equivalence ratio $\phi$ is increased, the intensity of flow oscillations is increased first and then decayed. The maximum $\mathrm{p}_{\mathrm{rms}}$ occurs at $\Phi=1.1$ and $\mathrm{V}_{\mathrm{f}}=5.0 \mathrm{~L} / \mathrm{min}$.

Detailed insights on the combustion-driven fluctuations are obtained by conducting autocorrelation of the measured pressure signal. This is shown in Fig. 6. It can be seen that at $\Phi=1.2$ and the methane volume flow rate is set to $\mathrm{V}_{\mathrm{f}}=2.0 \mathrm{~L} / \mathrm{min}$, the autocorrelation coefficient is so low as indicated by the red dots in Fig. 6(e). It reveals that there are no limit cycle oscillations present in the swirl combustor. However, as the methane volume flow rate $\mathrm{V}_{\mathrm{f}}$ is increased, limit cycle oscillations are generated. Furthermore, the oscillation frequency corresponding to $\mathrm{V}_{\mathrm{f}}=5.0 \mathrm{~L} / \mathrm{min}$ is relatively lower than that of $\mathrm{V}_{\mathrm{f}}=4.0 \mathrm{~L} / \mathrm{min}$, as illustrated in the wave-like form of the autocorrelation curve. As the methane flow rate is set to $\mathrm{V}_{\mathrm{f}}=5.0 \mathrm{~L} / \mathrm{min}$ and $\phi$ is increased from 0.8 to 1.2 , the oscillation frequency is increased. Finally, as $1.0 \leq \phi \leq 1.1$ and the methane volume flow rate is increased from $V_{\mathrm{f}}=3.0 \mathrm{~L} / \mathrm{min}$ to $5.0 \mathrm{~L} /$ min, the period is reduced and the dominant frequency is increased. This finding is consistent with our previous frequency spectrum analysis as shown in Fig. 4.

The recurrence plot (RP) is an effective tool for analyzing dynamical systems. It is a straightforward visualization of the recurrence matrix. The RP is applicable to time trace data in order to illustrate temporal correlations. The system dynamics can be approximately presented by a reconstruction of the phase space trajectory. The PR is an array of dots in a square. The values 0 and 1 in the matrix can be visualized by the black dot and white dot on the RP. Such as RP illustrates characteristic patterns, which is caused by typical dy- namical behavior. The recurrence plots for combustion-driven acoustic fluctuations in the present and absence of thermoacoustic instability are shown in Fig. 7(a) and (b) respectively. It can be seen from Fig. 7(a) that along the diagonal direction, there are equally-spaced diagonally-aligned line segments. They indicate the presence of periodic oscillations. This phenomena is not observed in Fig. 7(b). However, a higher density of recurrence points is present in some regions of Fig. 6(b). It indicates that these regions are dominated by background noise and high frequency dynamics. And there is a lack of deterministic structures.

The swirl combustor loses its stability and is associated with limit cycle oscillations at different frequencies, as $\phi=1.1$ and the fuel volume flow rate $V_{\mathrm{f}}$ is increased. The temperature contour along the axial direction of the swirl combustor is measured as shown in Fig. 8. It can be seen that as the fuel flow volume rate $V_{\mathrm{f}}$ is increased, the maximum temperature are increased. And the regions corresponding to maximum temperature are expanded.

Note that combustion instability may occur in practical swirl combustors, which are applied in gas turbines [44-46]. Such instability is characterized by large-amplitude harmonic or non-harmonic acoustic tones. Thus combustion instability can cause costly and catastrophic damage to the engine systems $[44,45]$. To ensure the combustion system being operated stably, a real-time monitoring system is applicable. This is typically done by implementing an acoustic pressure sensor, as we did in the present research. Furthermore, the real-time pressure measurement and the corresponding frequency spectrum analysis reveal that the transition from stable to unstable operation can occur in 

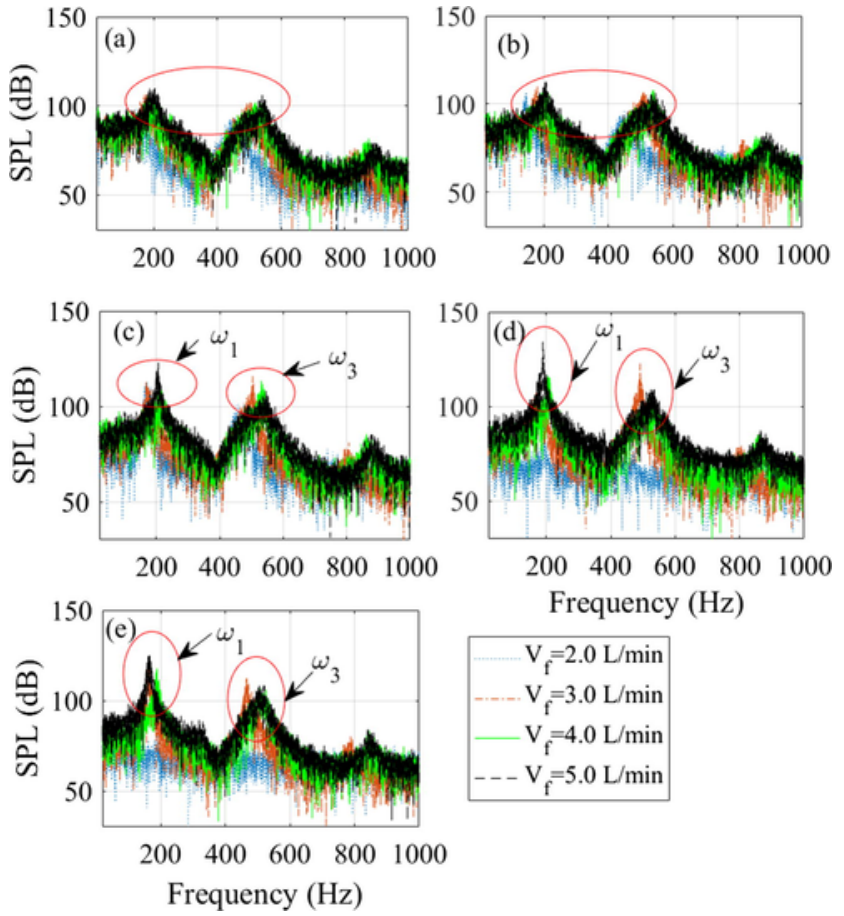

Frequency $(\mathrm{Hz})$

Fig. 4. Frequency spectrum of the measured pressure signal from the swirl combustor, as the methane volume flow rate $\mathrm{V}_{\mathrm{f}}$ is set to 4 different values: (a) $\phi=0.8$, (b) $\phi=0.9$, (c) $\phi=1.0,(\mathrm{~d}) \phi=1.1$ and $(\mathrm{e}) \phi=1.2$.
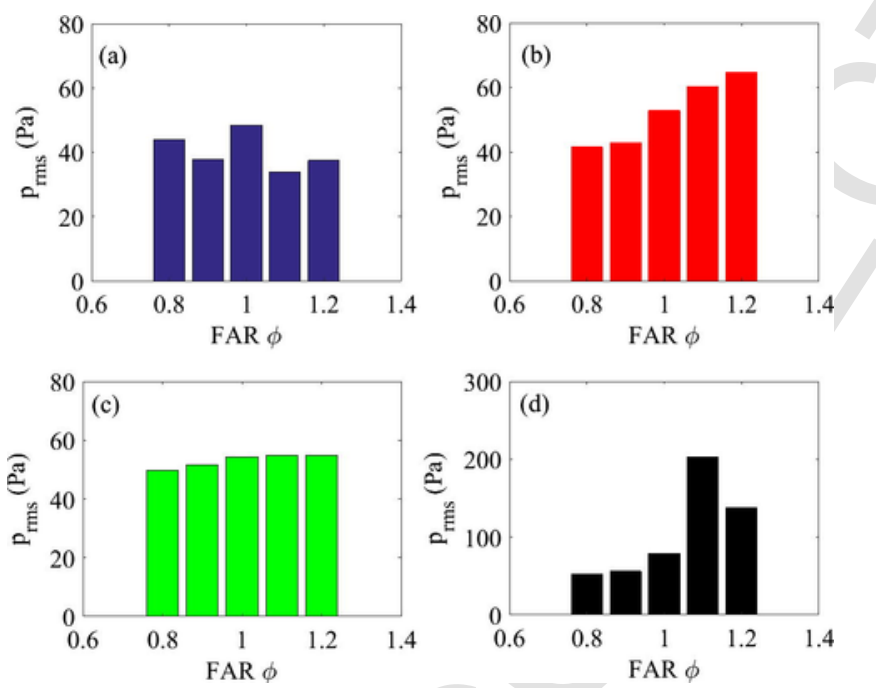

Fig. 5. Variation of the root mean square of the measured pressure with the fuel-air equivalence ratio (FAR), as the methane volume flow rate $V_{f}$ is set to 4 different values. (a) $\mathrm{V}_{\mathrm{f}}=2.0 \mathrm{~L} / \mathrm{min}$, (b) $\mathrm{V}_{\mathrm{f}}=3.0 \mathrm{~L} / \mathrm{min}$, (c) $\mathrm{V}_{\mathrm{f}}=4.0 \mathrm{~L} / \mathrm{min}$ and (d) $\mathrm{V}_{\mathrm{f}}=5.0 \mathrm{~L} / \mathrm{min}$.

the present swirling combustor. This is consistent with the finding obtained from a gas turbine combustor with a bluff-body applied [47]. Another common feature between the gas turbine combustor and the current swirling combustor is that hysteresis, nonlinear dynamics and limit cycle oscillations are experimentally observed in both types of combustion systems.

It is generally believed that there are no such combustion instability in the rich combustion mode, i.e. fuel-air ratio $\phi>1.0$. However, the present experimental work reveals that combustion-excited limit cycle oscillations can be generated as $\Phi \geq 1$.1. Furthermore, as the methane flow rate $V_{f}$ is increased, the dominant eigen-frequency transition from its harmonic at $\omega_{3}$ to the fundamental mode at $\omega_{1}$ is clearly observed. To the best knowledge of the authors,
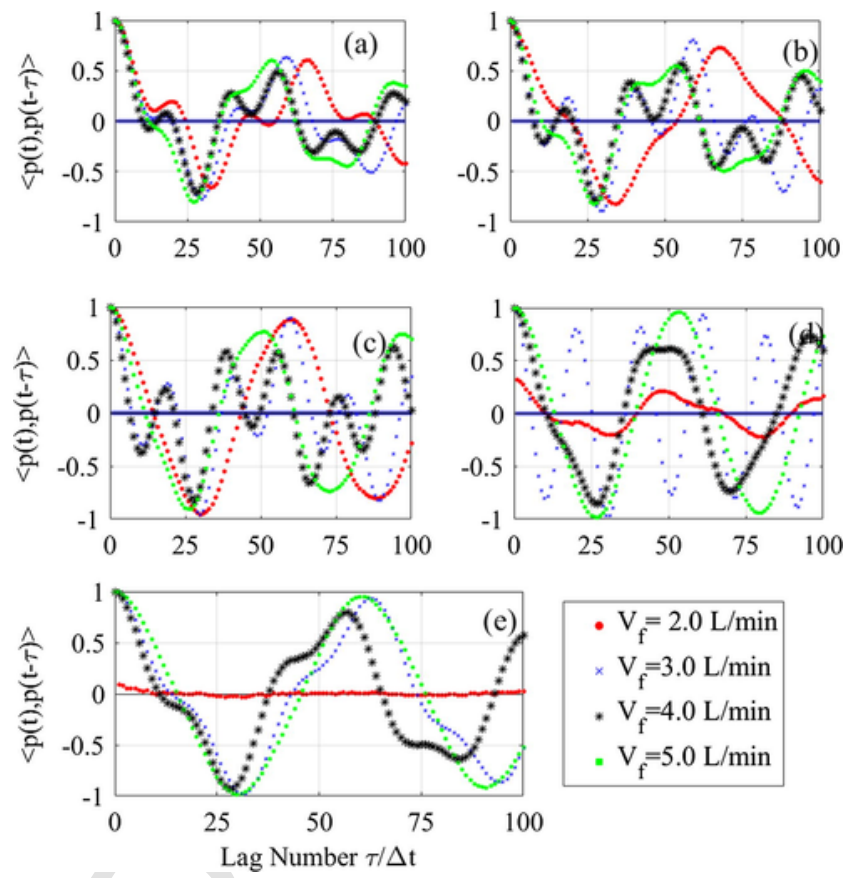

$\cdot \mathrm{V}_{\mathrm{f}}=2.0 \mathrm{~L} / \mathrm{min}$
$\times \mathrm{V}_{\mathrm{f}}=3.0 \mathrm{~L} / \mathrm{min}$
$. \mathrm{V}_{\mathrm{f}}=4.0 \mathrm{~L} / \mathrm{min}$
$. \mathrm{V}_{\mathrm{f}}=5.0 \mathrm{~L} / \mathrm{min}$

Fig. 6. Variation of the autocorrelation $\langle p(t), p(t-\tau)\rangle$ of the measured pressure with the delayed samples number, as the methane volume flow rate $V_{\mathrm{f}}$ is set to 4 different values. (a) $\phi=0.8$, (b) $\phi=0.9$, (c) $\phi=1.0$, (d) $\phi=1.1$ and (e) $\phi=1.2$.

such mode switching has not been reported before in such swirling combustion system [48-51].

\section{Conclusions}

In this work, the effects of fuel and air volume flow rates on producing self-excited nonlinear thermoacoustic oscillations are experimentally investigated. For this, a swirling thermoacoustic combustor is designed and experimentally tested. The dynamics characteristics of the nonlinear combustion system is quantified by measuring a pressure signal, as the methane volume flow rate $V_{f}$ is set to 4 different values. For each given fuel flow rate, the equivalent fuel-air ratio $\phi$ is varied from 0.8 to 1.2 . That is to say the combustion condition is changed from lean to rich combustion step by step. Note that industrial combustors are generally operated in lean combustion mode. However, the operating condition change does occur frequently. This may lead to the combustion mode being operated in rich condition. Thus we systematically measure the onset of combustion instability to examine the effects of (1) the fuel-air ratio and (2) the fuel flow rate. In addition, real-time acoustic measurement is found to play an important role in monitoring the conditions of the combustion system and combustion instability analysis.

When the fuel flow rate $V_{f}$ is low, there are no periodic self-sustained combustion oscillations produced. However, as the fuel flow rate $\mathrm{V}_{\mathrm{f}}$ is increased, limit cycle thermoacoustic oscillations are observed, depending on the volume flow rate of the fuel $V_{f}$ or the equivalence ratio $\phi$. As thermoacoustic instability occurs, the sound pressure level can be as high as $135 \mathrm{~dB}$. Furthermore, as the methane flow rate $V_{\mathrm{f}}$ is increased, the dominant eigen-frequency transition from its harmonic at $\omega_{3}$ to the fundamental mode at $\omega_{1}$ is clearly observed. This indicates the presence of hysteresis. The presence of harmonic peaks in the measured pressure spectrum reveals that the combustion system is highly nonlinear. In addition, increasing the fuel flow rate $V_{f}$ leads to the frequency $\omega_{1}$ of combustion-excited oscillations being increased by approximately $20 \%$. And the amplitude of the oscillations is increased. These findings are quite insightful for designing a feedback controller to stabilize a swirling combustion system. The present investigation characterizes the important roles of the volume flow rates of the fuel $V_{f}$ and the equivalence ratio $\phi$ on generating nonlinear thermoacoustic instability in a swirl combustor. The real-time data analyzing 

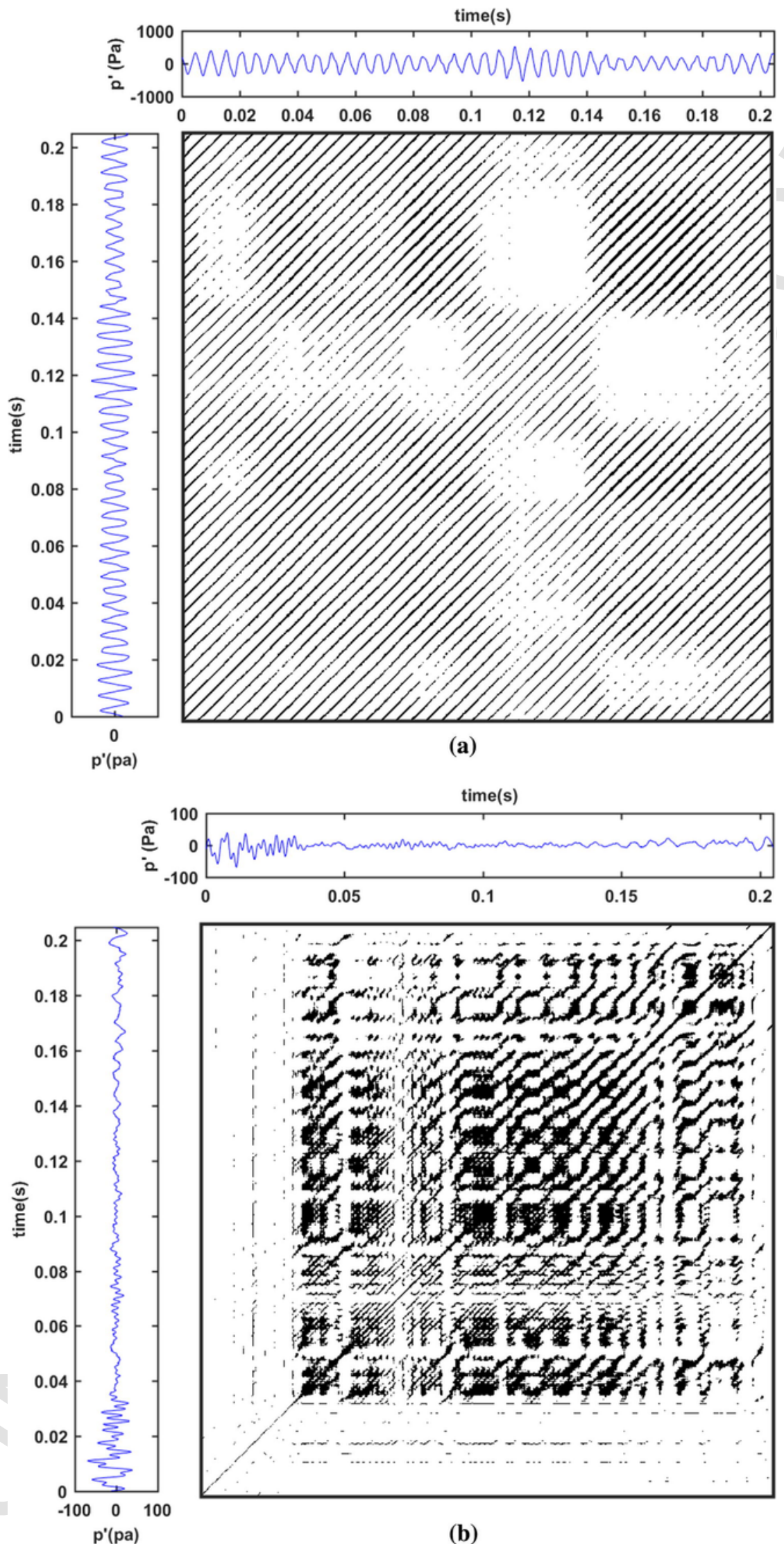

(b)

Fig. 7. Recurrence plots corresponding to the measure pressure signals at different conditions: (a) $V_{f}=2.0 \mathrm{~L} / \mathrm{min}$ and $V_{a}=23.8 \mathrm{~L} / \mathrm{min}$, (b) $V_{f}=5.0 \mathrm{~L} / \mathrm{min}$ and $\mathrm{V}_{\mathrm{a}}=40.0 \mathrm{~L} / \mathrm{min}$. Embedding dimension $M=3, \varepsilon=0.08$ (a), $\varepsilon=0.17$ (b). 


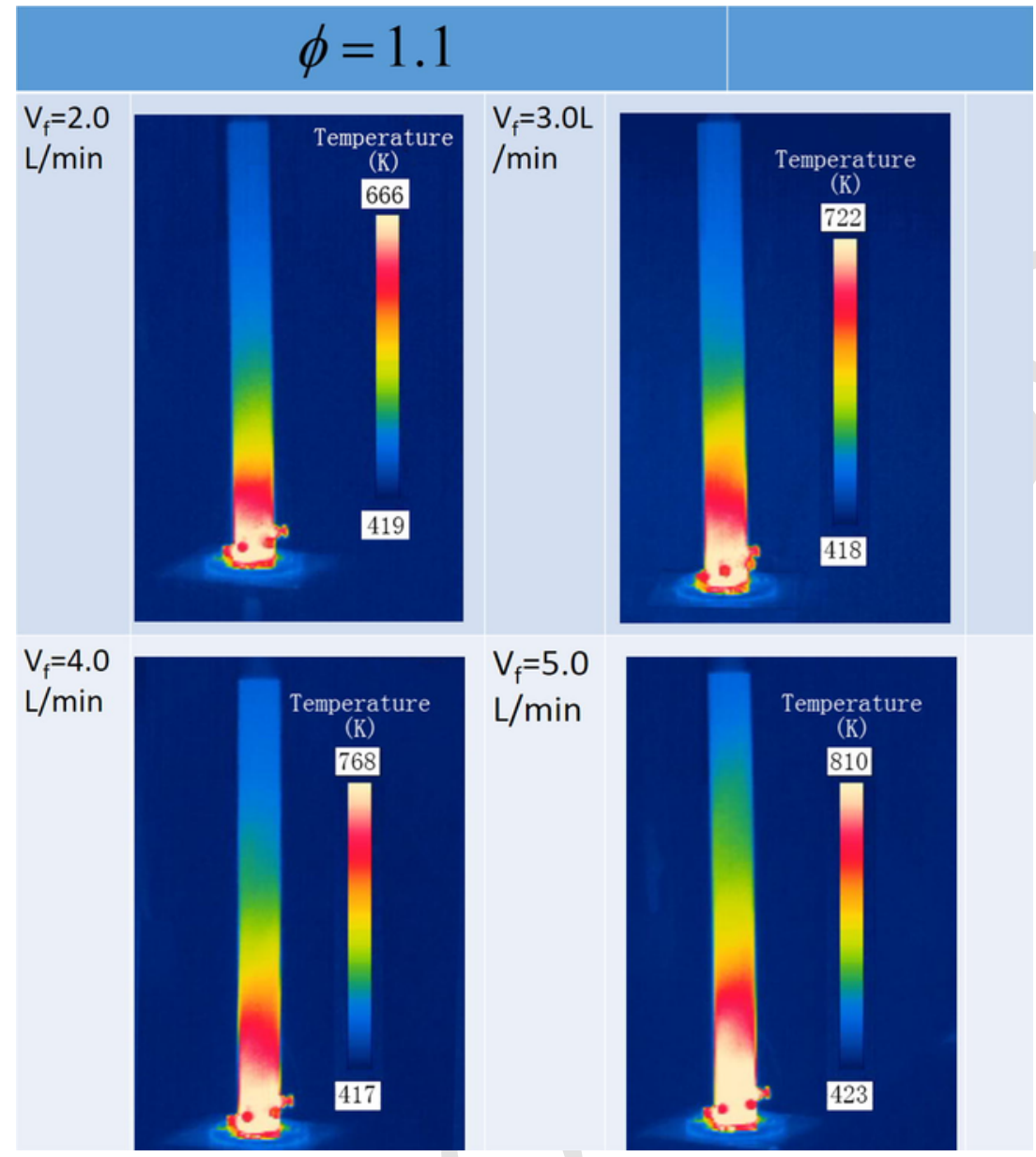

Fig. 8. Measured temperature contours along the axial direction of the combustor by using the infrared thermal imaging camera, as $\mathrm{V}_{\mathrm{f}}$ is set to 4 different values and $\phi=1.1$.

method and acoustic pressure measurements are also applicable to various combustion systems. And it opens up an applicable means to design a stable swirl combustor.

\section{Acknowledgements}

This work is supported by the University of Canterbury (New Zealand), Zhejiang University of Science and Technology (China), and Jiangsu
University of Science and Technology (China). This financial support by National Natural Science Foundation of China Grant Nos. 51206148, $51476145,51476146,11661141020,51776188$ and 51506079 is gratefully acknowledged. The experimental tests were conducted in Zhejiang University of Science and Technology by H. Zhao, S. Li and Z. Lu from Jiangsu University of Science and Technology, under the help and guidance of Prof. G. Li, Z. Zhang and Y. Zheng. The corresponding author conceived the idea, coordi-
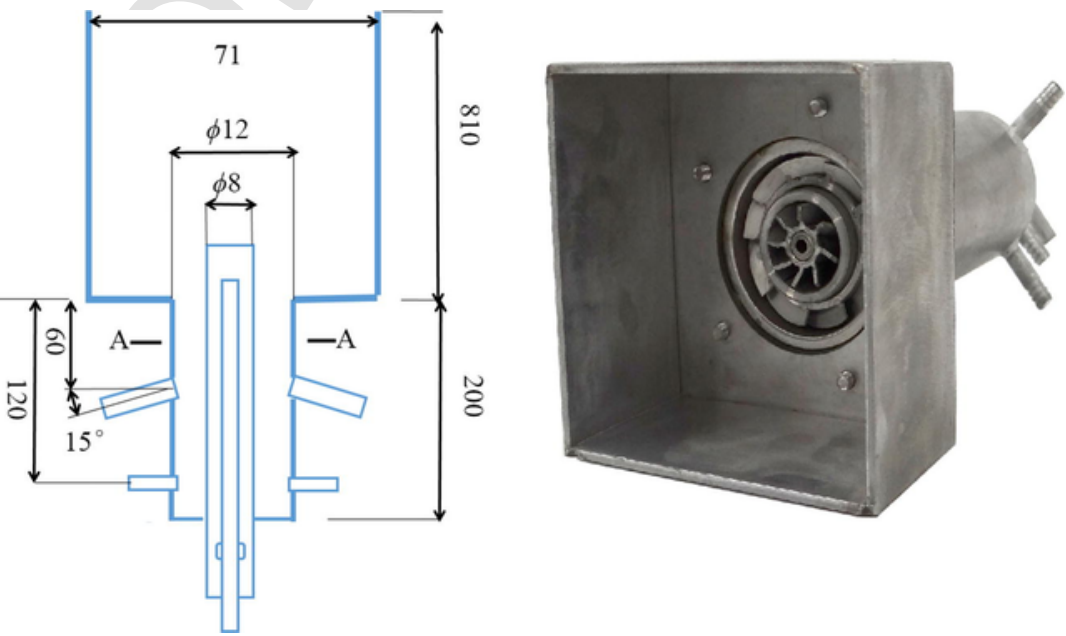

Fig. A1. Schematic drawing and photo of the swirling combustor with air and fuel injected. 
nated and initialized the research. Prof. D. Sun and W. Yang contributed to data-processing.

\section{Appendix A}

The schematic drawing and photo of the swirling combustion by using swirl vanes is shown in the graph below (see Fig. A1).

\section{References}

[1] S. Backhaus, G. Swift, A thermoacoustic Stirling heat engine, Nature 399 (1999) 335-338.

[2] A. Fichera, C. Losenno, A. Pagano, Experimental analysis of thermo-acoustic combustion instability, Appl Energy 70 (2001) 179-191.

[3] L. Cammarata, A. Fichera, A. Pagano, Neural prediction of combustion instability, Appl Energy 72 (2002) 513-528.

[4] A. Singh, M. Yu, A. Gupta, K. Bryden, Thermo-acoustic behavior of a swirl stabilized diffusion flame with heterogeneous sensors, Appl Energy 106 (2013) 1-16.

[5] A.P. Dowling, S.R. Stow, Acoustic analysis of gas turbine combustors, J Propul Pow 19 (2003) 751-764.

[6] A. Fichera, A. Pagano, Monitoring combustion unstable dynamics by means of control charts, Appl Energy 86 (2009) 1574-1581.

[7] G. Yu, X. Wang, W. Dai, E. Luo, Study on energy conversion characteristics of a high frequency standing-wave thermoacoustic heat engine, Appl Energy 111 (2013) 1147-1151.

[8] Z. Wu, Y. Chen, W. Dai, E. Luo, D. Li, Investigation on the thermoacoustic conversion characteristic of regenerator, Appl Energy 152 (2015) 156-161.

[9] T. Yazaki, S. Sugioka, F. Mizutani, H. Mamada, Nonlinear dynamics of a forced thermoacoustic oscillation, Phys Rev Lett 64 (1990) 2515.

[10] J. O'Connor, V. Acharya, T. Lieuwen, Transverse combustion instabilities: acoustic, fluid mechanic, and flame processes, Prog Energy Combust Sci 49 (2015) 1-39.

[11] Z. Yu, A.J. Jaworski, S. Backhaus, Travelling-wave thermoacoustic electricity gener ator using an ultra-compliant alternator for utilization of low-grade thermal energy, Appl Energy 99 (2012) 135-145.

[12] E.A. Gopalakrishnan, Y. Sharma, T. John, P.S. Dutta, R.I. Sujith, Early warning signals for critical transitions in a thermoacoustic system, Sci Rep 6 (2016) 35310.

[13] Y. Okuno, M. Small, H. Gotoda, Dynamics of self-excited thermoacoustic instability in a combustion system: pseudo-periodic and high-dimensional nature, Chaos 25 (2015) 043107

[14] J.P. Eckmann, S.O. Kamphorst, D. Ruelle, Recurrence plots of dynamical systems, Europhys Lett 4 (9) (1987) 973.

[15] J.P. Zbilut, A. Giuliani, C.L. Webber, Recurrence quantification analysis and principle components in the detection of short complex signals, Phys Lett A 237 (1998) 131-135.

[16] N. Marwan, J. Kurths, Line structures in recurrence plots, Phys Lett A 336 (2005) 349-357.

[17] N. Marwan, N. Wessel, U. Meyerfeldt, A. Schirdewan, J. Kurths, Recurrence plot based measures of complexity and its application to heart rate variability data, Phys Rev E 66 (2) (2002) 026702.

[18] H. Chiu, M. Summerfield, Theory of combustion noise, Acta Astronaut 1 (7-8) (1974) 967-984

[19] S. Candel, Durox, S. Ducruix, Birbaud, N. Noiray, T. Schuller, Flame dynamics and combustion noise: progress and challenges, Int J Aeroacoust 8 (1) (2009) 1-56.

[20] A.P. Dowling, Y. Mahmoudi, Combustion noise, Proc Combust Inst 35 (1) (2015) $1-65$.

[21] W.C. Strahle, J.I. Jagoda, Fractal image compression of Rayleigh, Raman, LIF and LDV data in turbulent reacting flows, Symp (Int)Combust, [Proc] 22 (1) (1989) 561.

[22] M.K. Ramachandra, W.C. Strahle, Acoustic signature from flames as a combustion diagnostic tool, AIAA J 21 (8) (1983) 1107-1114.

[23] W.C. Strahle, Turbulent combustion data analysis using fractals, AIAA J 29 (3) (1991) 401-409.

[24] K.R. Sreenivasan, R.A. Antonia, Annu. rev. The phenomenology of small-scale turbulence, Fluid Mech 29 (1) (1997) 429-435.
[25] F.C. Gouldin, K.N.C. Bray, J.Y. Chen, Chemical closure model for fractal flamelets, Western States Sect Combust Inst Paper (1988) 88-137.

[26] H. Kantz, T. Schreiber, Nonlinear time series analysis, Cambridge Press, 2004.

[27] H.D.I. Abarbanel, R. Brown, J.B. Kadtke, Prediction in chaotic nonlinear systems: methods for time series with broadband Fourier spectra, Phys Rev A 41 (4) (1990) 1782.

[28] L. Kabiraj, A. Saurabh, P. Wahi, R.I. Sujith, Route to chaos for combustion instability in ducted laminar premixed flames, Chaos 22 (2) (2012) 023129.

[29] J.D. Sterling, Nonlinear analysis and modelling of combustion instabilities in a lab oratory combustor, Combust Sci Technol 89 (1993) 159-167.

[30] M. Gorman, M. el Hamdi, B. Pearson, K.A. Robbins, Identification of intermittent ordered patterns as heteroclinic connections, Phys Rev Lett 76 (1996) 2061-2064

[31] H. Gotoda, T. Miyano, I.G. Shepherd, Dynamic properties of unstable motion of swirling premixed flames generated by a change in gravitational orientation, Phys Rev E 81 (2) (2010) 1-10.

[32] V. Nair, R.I. Sujith, A reduced-order model for the onset of combustion instability: physical mechanisms for intermittency and precursors, J Fluid Mech 747 (2014) 635-645.

[33] S. Domen, H. Gotoda, T. Kuriyama, Y. Okuno, S. Tachibana, Detection and prevention of blowout in a lean premixed gas-turbine model combustor using the concep of dynamical system theory, Proc Combust Inst 35 (3) (2015) 3245.

[34] L. Kabiraj, A. Saurabh, N. Karimi, A. Sailor, E. Mastorakos, A.P. Dowling, et al., Chaos in an imperfectly premixed model combustor, Chaos 25 (2) (2015) 023101

[35] L.P. Yang, S.L. Ding, G. Litak, E.Z. Song, X.Z. Ma, Identification and quantification analysis of nonlinear dynamics properties of combustion instability in a diesel engine, Chaos 25 (1) (2015) 013105.

[36] J.P. Eckmann, D. Ruelle, Ergodic theory of chaos and strange attractors, Rev Mod Phys 57 (3) (1985) 617.

[37] A. Brandstaeter, J. Swift, H.L. Swinney, A. Wolf, J.D. Farmer, E. Jen, et al., Low-dimensional chaos in a hydrodynamic system, Phys Rev Lett 51 (1983) 1442.

[38] K.R. Sreenivasan, Fractals and multifractals in fluid turbulence, Annu Rev Fluid Mech 23 (1) (1991) 539.

[39] S. Candel, D. Durox, T. Schuller, J.F. Bourgouin, J.P. Moeck, Dynamics of swirling flames, Annu Rev Fluid Mech 46 (2014) 147-173.

[40] X. Li, D. Zhao, Transient growth of acoustical energy associated with mitigating thermoacoustic oscillations, Appl Energy 169 (2016) 481-490.

[41] S. Li, Q. Li, L. Tang, B. Yang, J. Fu, C.A. Clarke, et al., Theoretical and experimetna demonstration of minimizing self-excited thermoacoustic oscillations by applying anti-sound technique, Appl Energy 181 (2016) 399-407.

[42] E. Jq, W. Zuo, X.L. Liu, Q.G. Peng, Y.W. Deng, H. Zhu, Effect of inlet pressure on wall temperature and exergy efficiency of the micro-cylindrical combustor with a step, Appl Energy 175 (2016) 337-345.

[43] E. Jq, X.H. Zhao, H.L. Liu, J.M. Chen, W. Zuo, Q.G. Peng, Field synergy analysis for enhancing heat transfer capability of a novel narrow-tube closed oscillating heat pipe, Appl Energy 175 (2016) 218-228.

[44] Y. Huang, V. Yang, Bifurcation of flame structure in a lean-premixed swirl-stabilized combustor: transition from stable to unstable flame, Combust Flame 136 (2004) 383-389.

[45] Y. Huang, V. Yang, Dynamics and stability of lean-premixed swirl-stabilized combustion, Prog Energy Combust Sci 35 (2009) 293-364.

[46] P. Palies, T. Schuller, D. Durox, S. Candel, Modeling of premixed swirling flames transfer functions, Proc Combust Inst 33 (2011) 2967-2974.

[47] T. Lieuwen, Experimental investigation of limit cycle oscillations in an unstable gas turbine combustor, J Propul Pow 18 (1) (2002) 61-67.

[48] A.E.E. Khalil, A.K. Gupta, Flame fluctuations in oxy-CO2-methane mixtures in swirl assisted distributed combustion, Appl Energy 204 (2017) 303-317.

[49] A. Valera-Medina, R. Marsh, J. Runyon, D. Pugh, P. Beasley, T. Hughes, et al., Ammonia-methane combustion in tangential swirl burners for gas turbine power generation, Appl Energy 185 (2017) 1362-1371.

[50] A.E.E. Khalil, A.K. Gupta, Acoustic and heat release signature for swirl assisted distributed combustion, Appl Energy 193 (2017) 125-138.

[51] A.K. Gupta, D.G. Lilley, N. Syred, Swirl flows, tunbridge wells, Abacus Press, Kent, 1984. 